\title{
Evaluation of RF power degradation in microwave photonic systems employing uniform period fibre Bragg gratings
}

\author{
G. Yu, W. Zhang and J. A. R. Williams
}

Photonics Research Group, Department of EECS, Aston University, Birmingham, B4 7ET, UK

\begin{abstract}
The authors investigate, the frequency
dependent RF power degradation in direct modulated microwave photonic systems employing uniform period fibre Bragg gratings (FBG) as reflective elements. The results have implications in terms of the available RF bandwidth and the stability requirements for the fibre Bragg gratings.
\end{abstract}

Index Terms - microwave photonics, fibre Bragg gratings

\section{INTRODUCTION}

Optical fibre techniques for the distribution and control of microwave signals within both communications and radar phased array antenna systems have incurred extensive research effort over the past decade [e.g. 1, 2, 3]. Fibre optic based true time delay (TTD) elements form a common element in many microwave photonic signal control architectures. Such TTD systems have been demonstrated using switched lengths of fibre [1], and dispersive fibre [4] or fibre Bragg grating (FBG) arrays with a tuneable optical carrier source [5]. The latter solution has advantages in

Email: yug@aston.ac.uk terms of being directly fibre compatible, more compact and simpler than the alternatives and is the one we are studying here. The conventional discrete fibre Bragg grating TTD line scheme is shown in Fig. 1. This optical signal is reflected from an array of Bragg gratings of differing resonant optical wavelengths. Tuning of the optical carrier selects a particular Bragg grating and hence the fibre length traversed and the group delay of the signal. The fibre Bragg grating is however not an ideal device in that it has a limited bandwidth and may introduce differing amplitudes and phase shifts between the sidebands of the signal and the optical carrier. Although this limitation induced by chirped fibre grating has been reported [6], the impact of uniform Bragg grating has not been studied in detail. In this paper, we investigate theoretically and experimentally the effects that these departures from the ideal have on the microwave signal.

\section{THEORY}

An ideal amplitude modulated optical signal will have two sidebands of equal amplitude and opposite phase relative to 
the optical carrier. Bragg gratings can give rise to

degradation in the received microwave signal amplitude by introducing changes in the relative amplitudes and phases of the sidebands. In Fig. 2 we plot a measured reflection and group delay responses for a uniform fibre Bragg grating (UFBG) of high reflectivity and broad bandwidth as would commonly be used in a true time delay application. The grating length is $5 \mathrm{~mm}$ with a coupling coefficient $\kappa=1300$ $\mathrm{m}^{-1}$. Provided the entire signal spectrum lies in the flat central reflectivity and time delay regions the microwave signal at the receiver will suffer only a delay. If however parts of the signal lie outside this central region there will be a differential power loss and across the spectrum, resulting in a degradation of the detected RF power at the optical receiver.

After reflection from a Bragg grating, the received electrical RF amplitude, generated by this optical signal can be expressed as $A_{R F}(\omega) \propto\left(R_{1} \cos \theta_{1}+R_{2} \cos \theta_{2}\right) \cos \left(\omega_{m} t\right)$

where $R_{1}, \theta_{1}$ and $R_{2}, \theta_{2}$ are the reflectivity and phase shifts corresponding to the upper and lower sidebands at microwave frequency $\omega_{\mathrm{m}}$. Thus, from the measured reflectivity and group delay characteristics of the grating we can calculate precisely the received RF amplitude as a function of optical carrier frequency and microwave RF frequency.

\section{EXPERIMENT}

The microwave response calculated using the expression (1) above was verified by comparing with the measured microwave amplitude spectral response for several different optical carrier wavelengths. The calibrated RF measurements were performed using a Photonetics Tunics1550 external cavity tuneable laser as the optical source with the HP8703A Lightwave component analyser (LCA) which combines the RF modulation and detection functions. The grating reflectivity spectra and group delay measurements, given in Fig. 2 were used in expression (1) to calculate the response for comparison. Both sets of results are plotted for different carrier wavelengths in Fig. 3. As can be seen we have good agreement between the RF frequency responses calculated from the grating spectra and those directly measured.

Fig. 3(a) shows the results taken when the carrier wavelength is $1541.3 \mathrm{~nm}$, in the centre of flat time delay response area. The two sidebands $( \pm 0.16 \mathrm{~nm}$ at $20 \mathrm{GHz}$ modulation) are all well within the response region. Therefore, the received RF response is flat at frequencies up to $20 \mathrm{GHz}$. In Fig. 3(b) the carrier wavelength is tuned to $1541.18 \mathrm{~nm}$. As the RF modulation frequency is increased, the lower sideband suffers an increasing delay relative to the carrier. At a frequency of $19 \mathrm{GHz}$, this delay corresponds to a $\pi$ phase shift and there is a null in the received RF spectrum. As the carrier wavelength is further decreased the RF frequency at which this null occurs moves to lower frequencies and the RF bandwidth of the grating is reduced. 
For example in Fig 3(c) with a carrier wavelength of $1541.165 \mathrm{~nm}$ has a null at a RF modulation frequency of 16GHz. Similar results are achieved if the carrier wavelength is shifted to longer wavelengths where the opposite sideband suffers an increasing delay. Differences between the two sets of curves are due to environmental changes between their measurement.

The nulls achieved are only about $8 \mathrm{~dB}$ deep and have oscillatory features on them. The depth of the nulls will depend on the relative strengths of the two sidebands when they are out of phase. If the two sidebands are of equal amplitude, they will entirely cancel out. In the results presented here, as a sideband approaches the edge of the grating spectrum its amplitude is reduced and its delay increased. If the opposite sideband retains constant amplitude then the sidebands will only partially cancel out for a $\pi$ phase change and the null depth will be reduced, as shown in Fig. 3. Additionally, the group delay characteristic of the grating is not itself a smooth function, particularly at the edges of the grating spectrum. Hence the relative delay in the sidebands does not vary smoothly and the RF spectrum demonstrates some oscillation.

Uniform gratings exhibit large sidelobes in the reflection spectra and oscillations in the group delay response. These features are undesirable in TTD applications and can be significantly suppressed by a suitable apodisation of the modulation depth of the Bragg grating along its length. In Fig. 4 we show the measured response for a $4.5 \mathrm{~mm}$ long grating with a raised cosine apodisation profile. The curves are much smoother and, compared with unapodised grating the time delay between central wavelength and edge of the grating has been greatly reduced. The microwave responses calculated from this profile and those directly measured are given in Fig. 5. As we can see, they are much flatter than those of the unapodised grating

This effect will certainly degrade the performance of a microwave photonics system employing such fibre gratings. If the unapodised fibre grating discussed above is used the available bandwidth of the system may go down to $16 \mathrm{GHz}$. The measurement of the microwave response of an optical fibre grating based notch filter was implemented to show the effect of the fibre grating on the filter's frequency response. The measured spectra are shown in Fig.6. As can be seen, Fig. 6(a) shows a normal notch response with the bandwidth over $20 \mathrm{GHz}$ where the unapodised grating was used and the carrier wavelength set at $1541.28 \mathrm{~nm}$; however, a distorted response is shown in Fig. 6(b) where the carrier wavelength was tuned to $1541.165 \mathrm{~nm}$. In Fig. 6(c) where the apodised grating was used and the carrier wavelength was set as that in Fig. 5(b), one can notice that, the amplitude and the visibility of the filter's response are slightly reduced as the microwave frequency increases and a nearly flat spectral response was obtained.

An important concern for the practical use of Bragg gratings in TTD elements is their sensitivity to temperature. The Bragg grating spectra shift in wavelength with temperature 
with a sensitivity of approximately $10^{-2} \mathrm{~nm} / \mathrm{K}$. For the apodised grating, reflecting a $20 \mathrm{GHz}$ microwave signal the bandwidth tolerated before there is a $3 \mathrm{~dB}$ drop is signal is $0.29 \mathrm{~nm}$. Thus, the Bragg grating has a useable temperature range of $29 \mathrm{~K}$ for a $3 \mathrm{~dB}$ change in signal power at $20 \mathrm{GHz}$.

\section{CONCLUSIONS}

We have described the evaluation of RF power degradation in microwave systems using UFBG as TTD elements.

Directly measured results show good agreement with those calculated from the grating spectra. Thus we can use a single measurement of the grating to determine precisely its performance in a microwave system. Unapodised and apodised gratings have been compared, with the apodised grating showing a significantly improved performance. We have used these measurements to determine the requirements on temperature stability for Bragg gratings used in microwave photonics systems.

\section{References}

[1]

W. Ng, A. A. Walston, G. L. Taggonan, J. J. Lee, I. L. Newberg, and N. Bernstein. "The first demonstration of an optically steered microwave phased array antenna using ture-time-delay", J. Lightwave Technol., Vol. 9, no. 9, pp.1124-1131, 1991.

[2] A. Seeds, "Optical technologies for phased array antennas", IEEE Trans, Electron., vol. E76-c, no.2, pp.198-206, 1995.

[3] I. Frigyes, and A. Seeds, "optically generated ture-time delay in phased array antennas", Trans, Microwave Theory and Techniques, vol. 43, no.9, pp.2378-2386, 1995
[4] R. D. Esman, M. Y. Frankel, J. L, Dexter, L. Godlberg, M. G. Parent, D. Stiwell, and D. G. Cooper, "Fibre-optic prism true timedelay antenna feed”, IEEE Photon. Technol. Lett., vol. 5, no.11, 1993

[5] A. Molony, L. Zhang, J. A. R. Williams, I. Bennion, "Fibre Bragg grating true time delay systems: Discrete grating array 3-bit delay lines and chirped grating 6-bit delay lines", IEEE Trans. Microwave Theory Tech. 45 (8), pp. 1527-1530, 1997

[6] W. Zhang and J. A. R. Williams, “Tunable fibre-optic notch filter using a dispersive device", Micrwave and Optical Tech. Lett. Vol. 22, No. 1., 1999 


\section{FIGURE CAPTIONS}

Fig. 1 An array of discrete uniform fibre Bragg gratings as a true time delay line.

Fig. 2 Measured reflectivity (a) and time delay response (b) of a uniform fibre Bragg grating.

Fig. 3 RF power against Microwave frequency. Solid lines are measured, dashed lines are calculated. (a) $\lambda_{c}=1541.3$ $\mathrm{nm}$, (b) $\lambda_{\mathrm{c}}=1541.18 \mathrm{~nm}$ (c) $\lambda_{\mathrm{c}}=1541.165 \mathrm{~nm}$.

Fig. 4 A measured reflectivity and time delay response of an apodised uniform Bragg grating.

Fig. 5 RF power against Microwave frequency. Solid lines are measured, dashed lines are calculated. (a) $\lambda_{\mathrm{c}}=1550.6$ $\mathrm{nm}$, (b) $\lambda_{\mathrm{c}}=1550.75 \mathrm{~nm}$.

Fig. 6 Measured frequency response of an optical fibre grating based notch filter. (a) with unapodised grating,

$\lambda_{\mathrm{c}}=1541.3 \mathrm{~nm}$, (b) with unapodised grating, $\lambda_{\mathrm{c}}=1541.18$ $\mathrm{nm}$, (c) with apodised grating, $\lambda_{\mathrm{c}}=1550.75 \mathrm{~nm}$ 


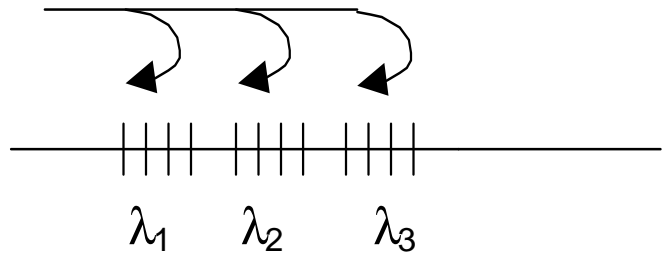

Fig. 1.

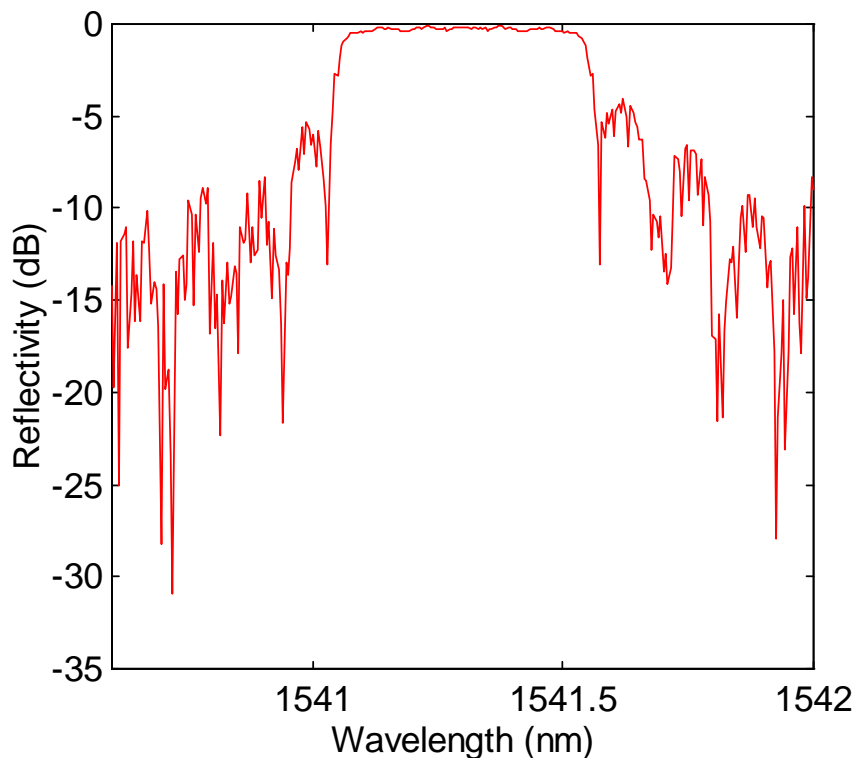

Fig. 2 (a)

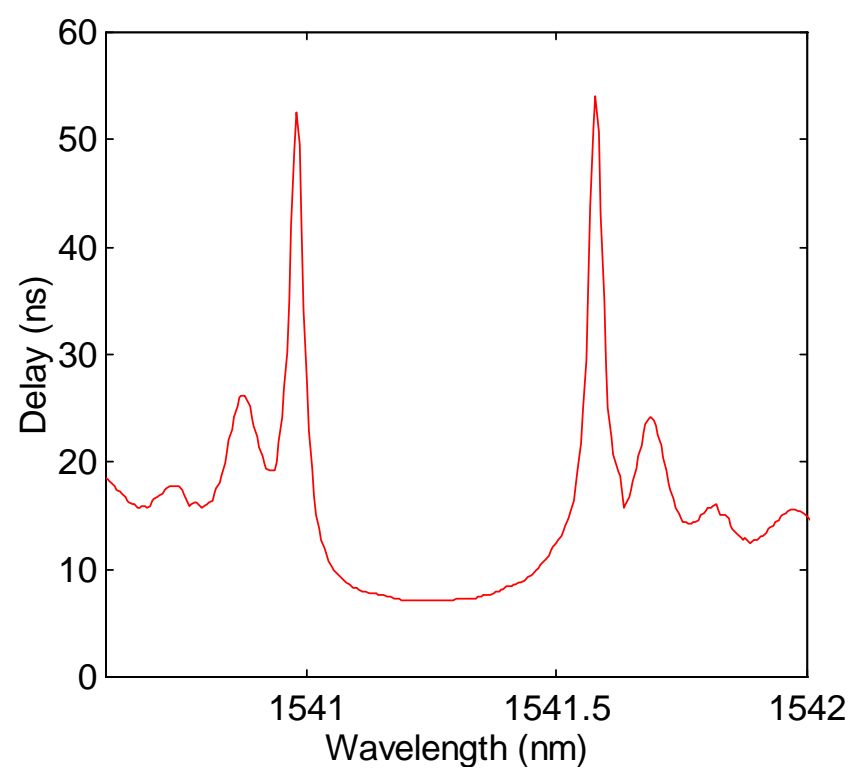

Fig. 2 (b) 

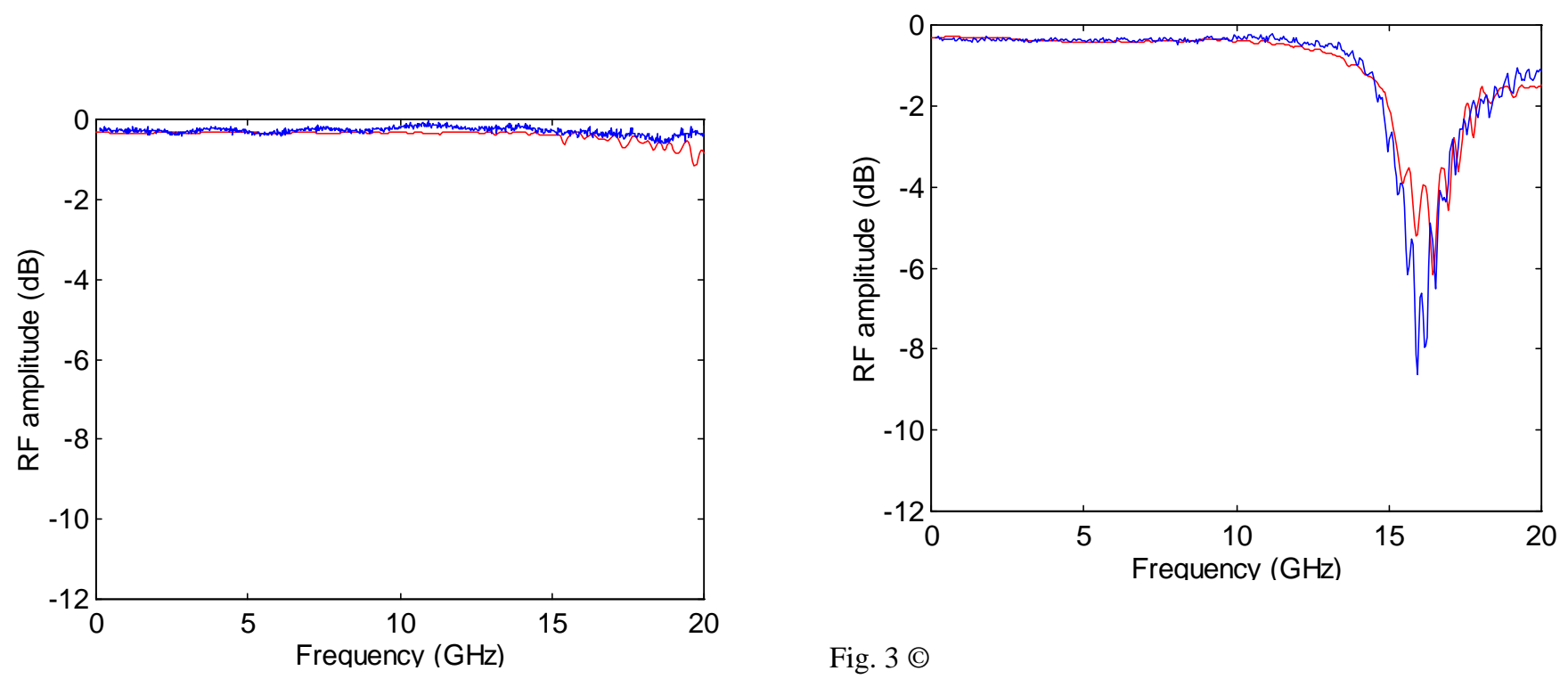

Fig. 3 ()

Fig. 3(a)

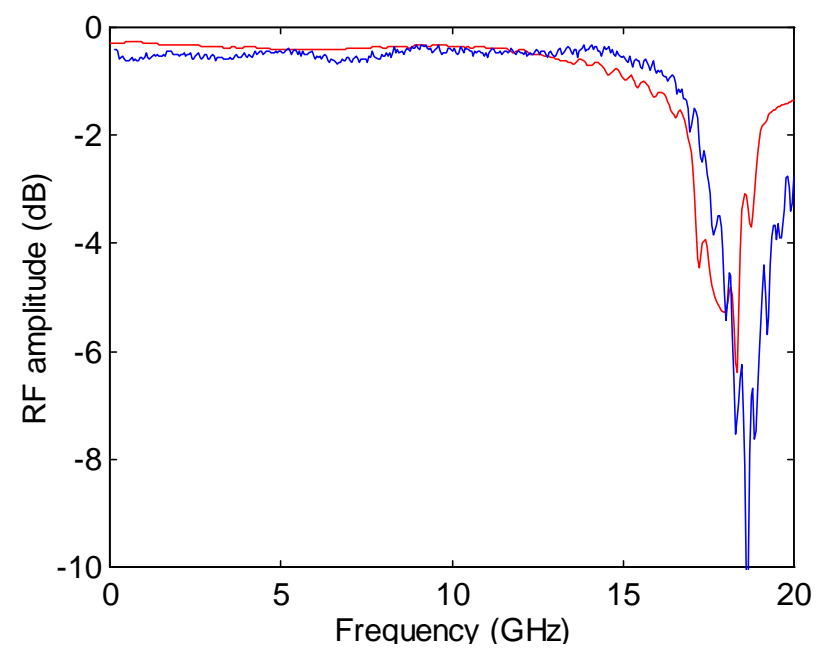

Fig. 3 (b) 



Fig 5 (a)

Fig. 4 (a)
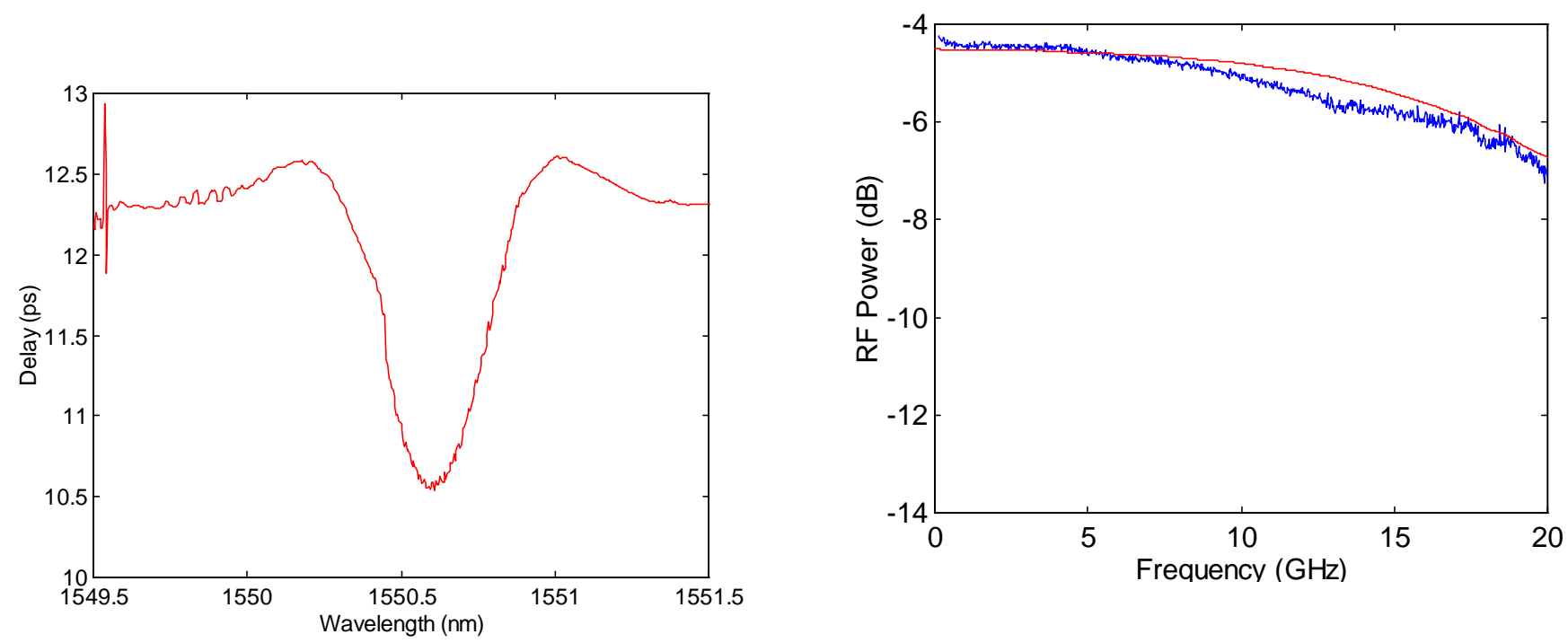

Fig 5 (b)

Fig 4 (b) 


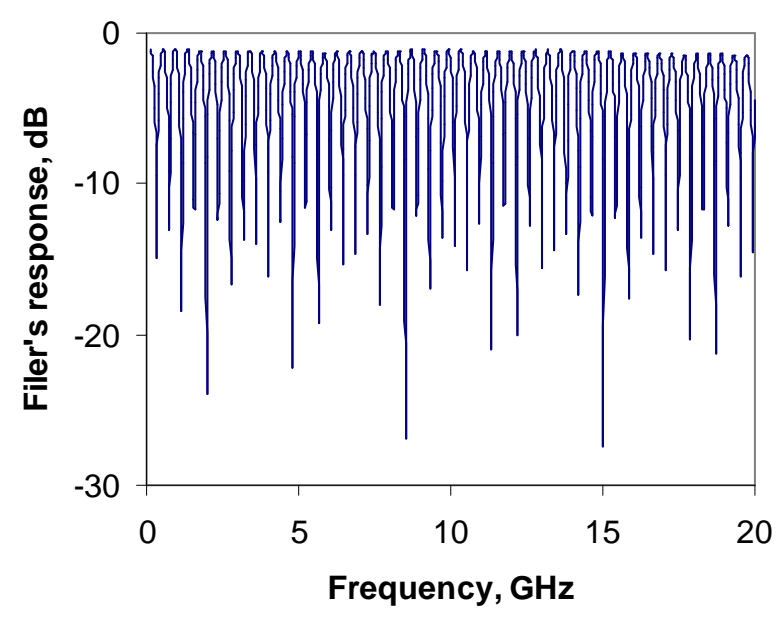

Fig. 6(a)

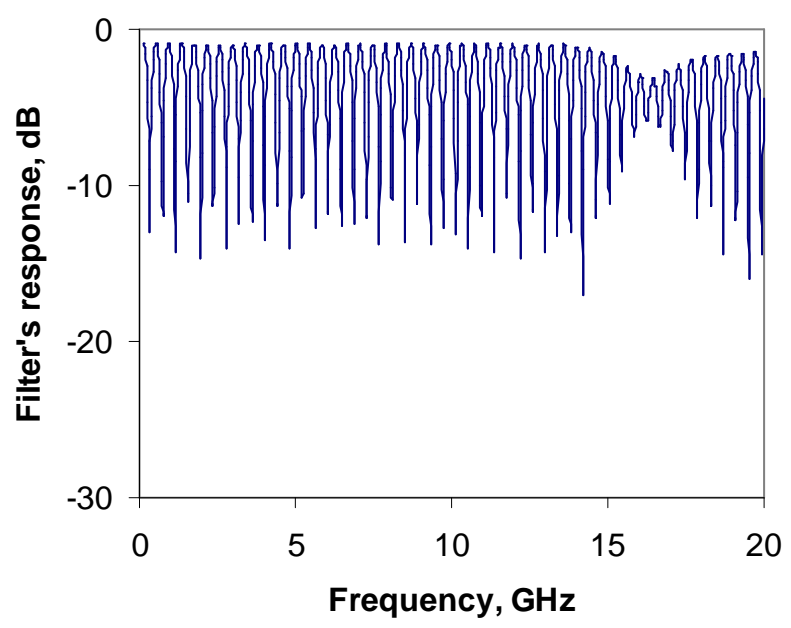

Fig. 6(b)

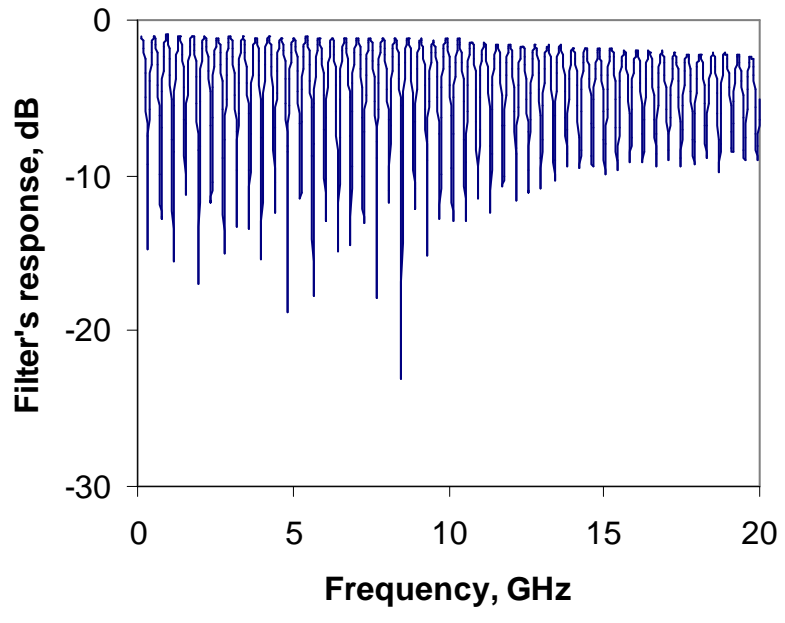

Fig. 6(c) 